\title{
On the Controllability for Second Order Hyperbolic Equations in Curved Polygons
}

W.D. BASTOS ${ }^{1}$, A. SPEZAMIGLIO ${ }^{2}$, Departamento de Matemática, IBILCE, UNESP, 15054-000 São José do Rio Preto, SP, Brasil.

\begin{abstract}
In this work we study exact boundary controllability for a class of hyperbolic linear partial differential equation with constant coefficient which includes the linear Klein-Gordon equation. We consider piecewise smooth domains on the plane, initial state with finite energy and control of Robin type, acting on the whole boundary or only on a part of it.
\end{abstract}

\section{Introduction}

The literature regarding exact boundary controllability for hyperbolic equations has increased enormously during the last three decades. Different methods have been developed by several authors. To mention some important contributions (for linear equations) we cite [1], [3-7]. Most of the results available in the literature consider domains with smooth boundary. It seems that [3] and [6] are the first results on boundary controllability dealing with domains with singularities on the boundary. In [2] and in the present work, we generalize some results of [3] and [6] by extending them to curved polygons and to the class of second order hyperbolic partial differential equations with constant coefficients which are reducible to the linear Klein-Gordon equation. More precisely, we consider exact controllability on the boundary for a hyperbolic partial differential equation $P(\partial) u=0$, in the independent variables $(x, y, t) \in \mathbb{R}^{2+1}$, where

$$
P(\partial)=\frac{\partial^{2}}{\partial t^{2}}-\frac{\partial^{2}}{\partial x^{2}}-\frac{\partial^{2}}{\partial y^{2}}+a_{0} \frac{\partial}{\partial t}+a_{1} \frac{\partial}{\partial x}+a_{2} \frac{\partial}{\partial y}+b,
$$

$a_{0}, a_{1}, a_{2}, b$ are real constants satisfying the inequality

$$
b-\frac{1}{4}\left\{a_{0}^{2}-a_{1}^{2}-a_{2}^{2}\right\} \geq 0 .
$$

Let $\Omega \subset \mathbb{R}^{2}$ be a bounded and simply connected domain with piecewise $C^{\infty}$ boundary $\partial \Omega$. We also assume that $\partial \Omega$ has no cusps and $\Omega$ lays in one side of $\partial \Omega$. We will refer to such a domain as a curved polygon. Let $\nu$ be the exterior

\footnotetext{
${ }^{1}$ waldemar@ibilce.unesp.br

${ }^{2}$ adalbert@ibilce.unesp.br
} 
unit vector normal to $\partial \Omega, \frac{\partial u}{\partial \nu}=\nabla u \cdot \nu$ and $B(\partial) u=\alpha u+\beta \frac{\partial u}{\partial \nu}$ with $\alpha^{2}+\beta^{2} \neq 0$. Throughout the paper, $H^{1}, H_{l o c}^{1}, H^{1 / 2}, L^{2}\left(=H^{0}\right)$, and $L_{l o c}^{2}\left(=H_{l o c}^{0}\right)$ denote the Sobolev spaces of square integrable functions with their usual norms. We will prove the following theorem.

Theorem 1.1. Let $\Omega \subset \mathbb{R}^{2}$ be a curved polygon. If the coefficients of $P(\partial)$ satisfy condition (1.1) then there exists $T>0$ such that, for every $\left(u_{0}, u_{1}\right) \in H^{1}(\Omega) \times$ $L^{2}(\Omega)$, there exists a control function $g \in L^{2}(\partial \Omega \times[0, T])$ so that the solution $u \in$ $H^{1}(\Omega \times[0, T])$ of the initial and boundary value problem

$$
\begin{array}{cl}
P(\partial) u=0 & \text { in } \Omega \times[0, T] \\
u(0)=u_{0}, \quad u_{t}(0)=u_{1} & \text { in } \Omega \\
B(\partial) u=g & \text { on } \partial \Omega \times[0, T]
\end{array}
$$

satisfies

$$
u(T)=u_{t}(T)=0 \quad \text { in } \Omega .
$$

If $\beta=0$, boundary condition (1.4) reads $\alpha u=g$. In this case, it will become clear from the proof of theorem 1 and the usual trace theorems that the control $g$ will be in $H^{1 / 2}(\partial \Omega \times[0, T])$.

Let $\Omega_{\infty}$ be the (open) angular sector $\Omega=\{(\rho \cos \theta, \rho \sin \theta): \rho>0,0<\theta<$ $\pi / m\}$, where $m$ is some positive integer. Let $\Omega$ be a curved polygon contained in the interior of $\Omega_{\infty}$, away from the origin and having some of its sides laying on the edges of the sector $\Omega_{\infty}$. Let $\Gamma_{o}$ be the part of the boundary laying on the edges of $\Omega_{\infty}$ and $\Gamma_{1}$ the remaining of $\partial \Omega$. Hence, $\Gamma_{o}$ is composed of straight segments while $\Gamma_{1}$ is the curved part of the boundary of $\Omega$. For such domain we consider the control problem with control action on the curved part of the boundary. We will prove the following theorem.

Theorem 1.2. Let $\Omega \subset \Omega_{\infty}$ be a curved polygon with some sides laying on the edges of $\Omega_{\infty}$ as described above. If the coefficients of $P(\partial)$ satisfy condition (1.1) then there exists $T>0$ such that, for every $\left(u_{0}, u_{1}\right) \in H^{1}(\Omega) \times L^{2}(\Omega)$, with $u_{0}=0$ on $\Gamma_{o}$, there exists a control function $g \in L^{2}\left(\Gamma_{1} \times[0, T]\right)$ so that the solution $u \in$ $H^{1}(\Omega \times[0, T])$ of the problem

$$
\begin{aligned}
P(\partial) u=0 & \text { in } \Omega \times[0, T] \\
u(0)=u_{0}, u_{t}(0)=u_{1} & \text { in } \Omega \\
u=0 \quad \text { on } \Gamma_{o} \times[0, T], \quad B(\partial) u=g & \text { on } \Gamma_{1} \times[0, T]
\end{aligned}
$$

satisfies

$$
u(T)=u_{t}(T)=0 \quad \text { in } \quad \Omega .
$$

This theorem extends the result of [2] to the equation $P(\partial) u=0$ and to a class of non-smooth domains a little bit larger than that considered in [2]. The Hilbert Uniqueness Method (HUM) introduced by J.-L. Lions in [4] is the most 
popular method used nowadays. That method imposes some restrictions on the geometry of the boundary which prevent its application to the domain considered in Theorem 2. This is a situation where the older methods present some advantages over the modern ones. We use the method introduced by D. L. Russell in [7] to prove theorems 1 and 2. A difficulty to apply it directly to the equation $P(\partial) u=0$ comes from the presence of the term $a_{0} \frac{\partial}{\partial t}$ which makes the equation not invariant under the change of variable $t=T-\tau$. This is the point where the restriction $b-\frac{1}{4}\left\{a_{0}^{2}-a_{1}^{2}-a_{2}^{2}\right\} \geq 0$ on the coefficients comes in. Lemma 1 below establishes the connection between Russell's procedure and the exact controllability problem for the equation $P(\partial) u=0$. A trace theorem due to D. Tataru [8] plays a significant role in the proof of the Theorems 1 and 2. It is worth noticing that the method used here lends itself very well to computations (finite difference) since only a pure initial value problem need to be computed.

\section{Preliminaries}

In this section we set up some tools to be used in the proof of the Theorems 1 and 2. Let $\Omega$ be a bounded domain of the plane. Let $a_{0}, a_{1}, a_{2}$ and $b$ be real numbers and $P(\partial)$ as in the previous section. Let $G(\partial)$ be the operator given by

$$
G(\partial)=\frac{\partial^{2}}{\partial t^{2}}-\frac{\partial^{2}}{\partial x^{2}}-\frac{\partial^{2}}{\partial y^{2}}+\lambda
$$

where $\lambda=b-\frac{1}{4}\left\{a_{0}^{2}-a_{1}^{2}-a_{2}^{2}\right\}$. Let $\gamma$ be an oriented $C^{\infty}$ arc in $\mathbb{R}^{2}$ with no self intersection.

Lemma 2.1. Let $T>0$ be such that every $\left(v_{o}, v_{1}\right) \in H^{1}(\Omega) \times L^{2}(\Omega)$ has an extension $\left(\widetilde{v}_{o}, \widetilde{v}_{1}\right) \in H^{1}\left(\mathbb{R}^{2}\right) \times L^{2}\left(\mathbb{R}^{2}\right)$, with compact support, so that the solution $\widetilde{v} \in H_{l o c}^{1}\left(\mathbb{R}^{2+1}\right)$ of

$$
\begin{array}{ll}
G(\partial) \widetilde{v}=0 & \text { in } \mathbb{R}^{2+1} \\
\widetilde{v}(0)=\widetilde{v}_{o} & \text { in } \mathbb{R}^{2} \\
\widetilde{v}_{t}(0)=\widetilde{v}_{1} & \text { in } \mathbb{R}^{2}
\end{array}
$$

satisfies

$$
\widetilde{v}(T)=\widetilde{v}_{t}(T)=0 \quad \text { in } \Omega .
$$

Then, every $\left(u_{0}, u_{1}\right) \in H^{1}(\Omega) \times L^{2}(\Omega)$ has an extension $\left(\widetilde{u}_{0}, \widetilde{u}_{1}\right) \in H^{1}\left(\mathbb{R}^{2}\right) \times L^{2}\left(\mathbb{R}^{2}\right)$, with compact support, so that the solution $\widetilde{u} \in H_{\text {loc }}^{1}\left(\mathbb{R}^{2+1}\right)$ of

$$
\begin{array}{ll}
P(\partial) \widetilde{u}=0 & \text { in } \mathbb{R}^{2+1} \\
\widetilde{u}(0)=\widetilde{u}_{0} & \text { in } \mathbb{R}^{2} \\
\widetilde{u}_{t}(0)=\widetilde{u}_{1} & \text { in } \mathbb{R}^{2}
\end{array}
$$

satisfies

$$
\widetilde{u}(T)=\widetilde{u}_{t}(T)=0 \quad \text { in } \Omega .
$$


Moreover, if $\widetilde{v}_{o}$ vanishes on $\gamma$ and $\widetilde{v}$ vanishes on $\gamma \times \mathbb{R}$, then the same holds true for $\widetilde{u}_{0}$ and $\widetilde{u}$ respectively on $\gamma$ and on $\gamma \times \mathbb{R}$.

Proof. The function $u$ satisfies the equation $P(\partial) u=0$ in $\mathbb{R}^{2+1}$ if and only if the function $v$ given by

$$
u=v \exp \left(-\frac{1}{2} a_{0} t+\frac{1}{2} a_{1} x+\frac{1}{2} a_{2} y\right)
$$

satisfies the equation $G(\partial) v=0$ in $\mathbb{R}^{2+1}$. Given any function $u_{0}$ and $u_{1}$ in $\Omega$ define

$$
\begin{gathered}
v_{0}=u_{0} \exp \left(-\frac{1}{2} a_{1} x-\frac{1}{2} a_{2} y\right), \\
v_{1}=\left[u_{1}+\frac{1}{2} a_{0} u_{0}\right] \exp \left(-\frac{1}{2} a_{1} x-\frac{1}{2} a_{2} y\right) .
\end{gathered}
$$

Suppose that there exist extensions $\widetilde{v}_{o}$ and $\widetilde{v}_{1}$ of $v_{o}$ and $v_{1}$ respectively, to the entire plane $\mathbb{R}^{2}$, both extension with compact support, and the solution $\widetilde{v}$ of $(2.1)-(2.4)$ satisfies $\widetilde{v}(T)=\widetilde{v}_{t}(T)=0$ in $\Omega$. The function $\widetilde{u}$ given by

$$
\widetilde{u}=\widetilde{v} \exp \left(-\frac{1}{2} a_{0} t+\frac{1}{2} a_{1} x+\frac{1}{2} a_{2} y\right)
$$

satisfies

$$
\begin{aligned}
& P(\partial) \widetilde{u}=0 \quad \text { in } \quad \mathbb{R}^{2+1}, \\
& \widetilde{u}(0)=\widetilde{v}_{o} \exp \left(\frac{1}{2} a_{1} x+\frac{1}{2} a_{2} y\right) \quad \text { in } \quad \mathbb{R}^{2}, \\
& \widetilde{u}_{t}(0)=\left[\widetilde{v}_{1}-\frac{1}{2} a_{0} \widetilde{v}_{o}\right] \exp \left(\frac{1}{2} a_{1} x+\frac{1}{2} a_{2} y\right) \quad \text { in } \quad \mathbb{R}^{2}, \\
& \widetilde{u}(T)=\widetilde{u}_{t}(T)=0 \quad \text { in } \quad \Omega .
\end{aligned}
$$

By the definition of $v_{o}$ and $v_{1}$ in terms of $u_{0}$ and $u_{1}$, we see that the restriction of $\widetilde{v}_{o} \exp \left(\frac{1}{2} a_{1} x+\frac{1}{2} a_{2} y\right)$ and $\left[\widetilde{v}_{1}-\frac{1}{2} a_{0} \widetilde{v}_{o}\right] \exp \left(\frac{1}{2} a_{1} x+\frac{1}{2} a_{2} y\right)$ to the set $\Omega$ are exactly the functions $u_{0}$ and $u_{1}$. If we define $\widetilde{u}_{o}=\widetilde{u}(0)$ and $\widetilde{u}_{1}=\widetilde{u}_{t}(0)$ we see that $\widetilde{u}_{o}$ and $\widetilde{u}$ are extensions of $u_{0}$ and $u_{1}$, respectively, to the plane, with compact support. Hence $\widetilde{u}, \widetilde{u}_{o}$ and $\widetilde{u}_{1}$ satisfy conditions $(2.6)-(2.9)$ as we wanted to prove. The other statement is obvious.

Lemma 2.2. Let $U \subset \mathbb{R}^{2}$ be a bounded domain and $f, g \in C_{o}^{\infty}\left(\mathbb{R}^{2}\right)$ be functions with compact support in $U$. Let $u \in C^{\infty}\left(\mathbb{R}^{2+1}\right)$ be the solution of the Cauchy problem for the equation $G(\partial) u=0$ with $\lambda \geq 0$ and initial data $u(0)=f, u_{t}(0)=g$. For each $T_{0}>\operatorname{diam}(U)$, there exists $K=K\left(\lambda, T_{0}, U\right)>0$ such that for every multiindex $\alpha=\left(\alpha_{1}, \alpha_{2}, \alpha_{3}\right) \in \mathbb{N}^{3}$, with $|\alpha|=\alpha_{1}+\alpha_{2}+\alpha_{2} \leq 1$ we have

$$
\left|\frac{\partial^{|\alpha|}}{\partial(x, t)^{\alpha}} u(x, t)\right| \leq \frac{K}{t}\left\{\|f\|_{H^{1}(U)}+\|g\|_{L^{2}(U)}\right\}
$$

for every $x \in U$ and $t \geq T_{0}$. 
Proof. For each $t>0$ the function $u$ is given by

$$
\begin{aligned}
u\left(x_{1}, x_{2}, t\right)= & \frac{1}{2 \pi} \int_{r<t} g\left(y_{1}, y_{2}\right) \frac{\cos \left(m \sqrt{t^{2}-r^{2}}\right)}{\sqrt{t^{2}-r^{2}}} d y_{1} d y_{2}+ \\
& +\frac{\partial}{\partial t}\left[\frac{1}{2 \pi} \int_{r<t} f\left(y_{1}, y_{2}\right) \frac{\cos \left(m \sqrt{t^{2}-r^{2}}\right)}{\sqrt{t^{2}-r^{2}}} d y_{1} d y_{2}\right],
\end{aligned}
$$

where $r=|x-y|=\sqrt{\left(y_{1}-x_{1}\right)^{2}+\left(y_{2}-x_{2}\right)^{2}}$ and $m=\sqrt{\lambda}$. Now observe that for each $x \in U$ and $t \geq T_{0}$ the ball with center $x$ and radius $t$ includes $U$ in its interior. Since the initial data have compact support in $U$ we can write

$$
\begin{aligned}
u\left(x_{1}, x_{2}, t\right)= & \frac{1}{2 \pi} \int_{U} g\left(y_{1}, y_{2}\right) \frac{\cos \left(m \sqrt{t^{2}-r^{2}}\right)}{\sqrt{t^{2}-r^{2}}} d y_{1} d y_{2}+ \\
& +\frac{\partial}{\partial t}\left[\frac{1}{2 \pi} \int_{U} f\left(y_{1}, y_{2}\right) \frac{\cos \left(m \sqrt{t^{2}-r^{2}}\right)}{\sqrt{t^{2}-r^{2}}} d y_{1} d y_{2}\right]
\end{aligned}
$$

for every $x=\left(x_{1}, x_{2}\right) \in U$ and $t>T_{0}$.

In order to estimate all the derivatives of $u$ listed in (2.10) it suffices to estimate the function $\zeta(x, y, t)=\frac{\cos \left(m \sqrt{t^{2}-r^{2}}\right)}{\sqrt{t^{2}-r^{2}}}$ and its partial derivatives $\frac{\partial}{\partial x_{1}}, \frac{\partial}{\partial x_{2}}, \frac{\partial}{\partial t}, \frac{\partial^{2}}{\partial x_{1} \partial t}$, $\frac{\partial^{2}}{\partial x_{2} \partial t}, \frac{\partial^{2}}{\partial t^{2}}$, uniformly for $x, y \in U$ and $t>T_{0}$. The symmetry between $x_{1}$ and $x_{2}$ reduces our work to estimate only $\zeta, \frac{\partial \zeta}{\partial x_{1}}, \frac{\partial \zeta}{\partial t}, \frac{\partial^{2} \zeta}{\partial x_{1} \partial t}$ and $\frac{\partial^{2} \zeta}{\partial t^{2}}$.

Let $\chi(s)=\frac{1}{\sqrt{1-s^{2}}}$. By direct computation we obtain

$$
\begin{aligned}
\zeta(x, y, t)= & \frac{1}{t} \chi\left(\frac{r}{t}\right) \cos \left(m t / \chi\left(\frac{r}{t}\right)\right), \\
\frac{\partial \zeta}{\partial x_{1}}(x, y, t)= & \frac{1}{t}\left\{m \chi\left(\frac{r}{t}\right)^{2} \frac{x_{1}-y_{1}}{t} \operatorname{sen}\left(m t / \chi\left(\frac{r}{t}\right)\right)\right. \\
& \left.+\frac{1}{t} \chi\left(\frac{r}{t}\right)^{3} \frac{x_{1}-y_{1}}{t} \cos \left(m t / \chi\left(\frac{r}{t}\right)\right)\right\} \\
\frac{\partial \zeta}{\partial t}(x, y, t)= & \frac{1}{t}\left\{-m \chi\left(\frac{r}{t}\right)^{2} \operatorname{sen}\left(m t / \chi\left(\frac{r}{t}\right)\right)-\frac{1}{t} \chi\left(\frac{r}{t}\right)^{3} \cos \left(m t / \chi\left(\frac{r}{t}\right)\right)\right\}, \\
\frac{\partial^{2} \zeta}{\partial x_{1} \partial t}(x, y, t)= & \frac{1}{t}\left\{m^{2} \chi\left(\frac{r}{t}\right)^{3} \frac{x_{1}-y_{1}}{t} \cos \left(m t / \chi\left(\frac{r}{t}\right)\right)\right. \\
& -\frac{3}{t^{2}} \chi\left(\frac{r}{t}\right)^{5} \frac{x_{1}-y_{1}}{t} \cos \left(m t / \chi\left(\frac{r}{t}\right)\right) \\
& \left.-\frac{3 m}{t} \chi\left(\frac{r}{t}\right)^{4} \frac{x_{1}-y_{1}}{t} \operatorname{sen}\left(m t / \chi\left(\frac{r}{t}\right)\right)\right\} \\
\frac{\partial^{2} \zeta}{\partial t^{2}}(x, y, t)= & \frac{1}{t}\left\{3 m \frac{1}{t} \chi\left(\frac{r}{t}\right)^{4} \operatorname{sen}\left(m t / \chi\left(\frac{r}{t}\right)\right)-m \frac{1}{t} \chi\left(\frac{r}{t}\right)^{2} \operatorname{sen}\left(m t / \chi\left(\frac{r}{t}\right)\right)\right. \\
& -\frac{1}{t^{2}} \chi\left(\frac{r}{t}\right)^{3} \cos \left(m t / \chi\left(\frac{r}{t}\right)\right)+\frac{3}{t^{2}} \chi\left(\frac{r}{t}\right)^{5} \cos \left(m t / \chi\left(\frac{r}{t}\right)\right) \\
& \left.-m^{2} \chi\left(\frac{r}{t}\right)^{3} \cos \left(m t / \chi\left(\frac{r}{t}\right)\right)\right\} .
\end{aligned}
$$


Observe that if $x, y \in U$ and $t \geq T_{0}$ then $\frac{r}{t} \leq \frac{\operatorname{diam}(U)}{T_{0}}<1$. Lets fix $\eta>0$ such that $\frac{\operatorname{diam}(U)}{T_{0}}<\eta<1$ and consider the function $\chi$ in the interval $[-\eta, \eta]$. If we define $K_{1}=\left(\frac{1}{\sqrt{1-\eta^{2}}}\right)^{5}$ we get $\chi\left(\frac{r}{t}\right)^{k} \leq K_{1}$, for every $x, y \in U, t>T_{0}$ and $k=1,2, \ldots, 5$. Now, observing that $\left|\frac{x_{1}-y_{1}}{t}\right| \leq \frac{r}{t}<\eta<1$ we obtain

$$
\begin{aligned}
|\zeta(x, y, t)| & \leq \frac{1}{t} K_{1} \\
\left|\frac{\partial \zeta}{\partial x_{1}}(x, y, t)\right| & \leq \frac{1}{t} K_{1}\left\{m+\frac{1}{T_{0}}\right\} \\
\left|\frac{\partial \zeta}{\partial t}(x, y, t)\right| & \leq \frac{1}{t} K_{1}\left\{m+\frac{1}{T_{0}}\right\} \\
\left|\frac{\partial^{2} \zeta}{\partial x_{1} \partial t}(x, y, t)\right| & \leq \frac{1}{t} K_{1}\left\{m^{2}+\frac{3 m}{T_{0}}+\frac{3}{T_{0}^{2}}\right\} \\
\left|\frac{\partial^{2} \zeta}{\partial t^{2}}(x, y, t)\right| & \leq \frac{1}{t} K_{1}\left\{\frac{4 m}{T_{0}}+\frac{3}{T_{0}^{2}}+\frac{1}{T_{0}^{3}}\right\}
\end{aligned}
$$

for every $x, y \in U$ and $t>T_{0}$.

For each $\alpha=\left(\alpha_{1}, \alpha_{2}, \alpha_{3}\right)$ with $|\alpha| \leq 1$ we differentiate (2.12), take absolute value on both sides and use the estimates above to obtain

$$
\left|\frac{\partial^{|\alpha|}}{\partial(x, t)^{\alpha}} u(x, t)\right| \leq \frac{K_{2}}{2 \pi t}\left\{\int_{U}|f(y)| d y+\int_{U}|g(y)| d y\right\}
$$

for every $x \in U$ and $t \geq T_{0}$, where $K_{2}$ is a constant depending on $m, K_{1}$ and $T_{0}$. To finish we use Cauchy-Schwartz inequality together with $\|f\|_{L^{2}} \leq\|f\|_{H^{1}}$ to obtain

$$
\left|\frac{\partial^{|\alpha|}}{\partial(x, t)^{\alpha}} u(x, t)\right| \leq \frac{K_{2}|U|^{\frac{1}{2}}}{2 \pi t}\left\{\|f\|_{H^{1}(U)}+\|g\|_{L^{2}(U)}\right\},
$$

where $|U|$ denotes the Lebesgue measure of $U$, uniformly in $x \in U$ and $t \geq T_{0}$. If we set $K=\frac{K_{2} \mid U^{\frac{1}{2}}}{2 \pi}$ we conclude the proof.

An immediate consequence of Lemma 2 is the inequality

$$
\left\|u_{t}(\cdot, t)\right\|_{L^{2}(U)}^{2}+\|u(\cdot, t)\|_{H^{1}(U)}^{2} \leq \frac{k}{t^{2}}\left\{\left\|u_{t}(\cdot, 0)\right\|_{L^{2}(U)}^{2}+\|u(\cdot, 0)\|_{H^{1}(U)}^{2}\right\}
$$

for every $t>T o>\operatorname{diam}(U)$. Here, $k$ is a constant depending on $\lambda, U$ and $T_{0}$.

Lemma 2.3. Let $\Omega \subset \mathbb{R}^{2}$ be a bounded domain and $\Omega_{\delta}$ be an open neighborhood of $\Omega$. Let $\left(\widetilde{v}_{o}, \widetilde{v}_{1}\right) \in H^{1}\left(\mathbb{R}^{2}\right) \times L^{2}\left(\mathbb{R}^{2}\right)$ be functions with compact support in $\Omega_{\delta}$. If $\lambda \geq 0$, there exists a constant $k>0$, independent of $\left(\widetilde{v}_{o}, \widetilde{v}_{1}\right)$, such that the solution $\widetilde{v} \in H_{l o c}^{1}\left(\mathbb{R}^{2+1}\right)$ of the Cauchy problem

$$
\begin{array}{cll}
G(\partial) \widetilde{v}=0 & \text { in } & \mathbb{R}^{2+1}, \\
\widetilde{v}(0)=\widetilde{v}_{o} & \text { in } & \mathbb{R}^{2}, \\
\widetilde{v}_{t}(0)=\widetilde{v}_{1} & \text { in } & \mathbb{R}^{2},
\end{array}
$$


satisfies

$$
\left\|\widetilde{v}_{t}(\cdot, t)\right\|_{L^{2}\left(\Omega_{\delta}\right)}^{2}+\|\widetilde{v}(\cdot, t)\|_{H^{1}\left(\Omega_{\delta}\right)}^{2} \leq \frac{k}{t^{2}}\left\{\left\|\widetilde{v}_{1}\right\|_{L^{2}\left(\Omega_{\delta}\right)}^{2}+\left\|\widetilde{v}_{0}\right\|_{H^{1}\left(\Omega_{\delta}\right)}^{2}\right\}
$$

for every $t>\operatorname{diam}\left(\Omega_{\delta}\right)$.

Proof. It suffices to observe that the solution $\widetilde{v} \in H_{l o c}^{1}\left(\mathbb{R}^{2+1}\right)$ is obtained as the limit of a sequence of smooth solution to the Cauchy problem with smooth initial data and use (2.13) for such sequence

We finish this section setting a result on the regularity of the traces of the solutions of the equation $P(\partial) u=0$ which will be needed to study the controllability problems considered here. We start explaining a result due to Tataru [8]. Let $L(\xi, D)$ be a hyperbolic partial differential operator, with $C^{\infty}$ coefficients depending on $\xi$ in some open set $\Xi \subset \mathbb{R}^{n}$. Let $\Sigma \subset \Xi$ be an oriented surface of class $C^{\infty}$, timelike with respect to the operator $L$. Let $\nu=\left(\nu_{1}, \ldots \nu_{n}\right)$ be the unit vector normal to $\Sigma$. If $\Sigma a^{i j} \frac{\partial^{2}}{\partial \xi_{i} \partial \xi_{j}}$ is the principal part of $L(\xi, D)$ then $\frac{\partial u}{\partial \nu}=\Sigma a^{i j} \frac{\partial u}{\partial \xi_{i}} \nu_{j}$ is the conormal derivative of $u$ with respect to $L(\xi, D)$ along $\Sigma$. In [8] Theorem 2 establishes that if $u \in H_{l o c}^{1}(\Xi)$ is such that $L(\xi, D) u \in L_{l o c}^{2}(\Xi)$ then $\frac{\partial u}{\partial \nu} \in L_{l o c}^{2}(\Sigma)$. Now we apply this result to the equation $P(\partial) u=0$. Let $\Xi$ be the space $\mathbb{R}^{2+1}$ and set $\Sigma=\gamma \times \mathbb{R}$ where $\gamma$ is a $C^{\infty}$ arc in $\mathbb{R}^{2}$ with no self intersection and unit normal vector $\nu=\left(\nu_{1}, \nu_{2}\right)$. If we take $L(\xi, D)=P(\partial)=\frac{\partial^{2}}{\partial t^{2}}-\frac{\partial^{2}}{\partial x^{2}}-\frac{\partial^{2}}{\partial y^{2}}+a_{0} \frac{\partial}{\partial t}+a_{1} \frac{\partial}{\partial x}+a_{2} \frac{\partial}{\partial y}+b$ and observe that $\left(\nu_{1}, \nu_{2}, 0\right)$ is the unit vector normal to $\Sigma$ we see that the conormal derivative of $u$ with respect to $P$ along $\Sigma$ is simply the usual normal derivative $\frac{\partial u}{\partial \nu}$. The following lemma is an immediate consequence of the mentioned Tataru's result and the usual trace theorems [9].

Lemma 2.4. Let $\widetilde{u} \in H_{l o c}^{1}\left(\mathbb{R}^{2+1}\right)$ be the solution for the equation $P(\partial) \widetilde{u}=0$ with initial data $\left(\widetilde{u}_{0}, \widetilde{u}_{1}\right) \in H^{1}\left(\mathbb{R}^{2}\right) \times L^{2}\left(\mathbb{R}^{2}\right)$. Let $\gamma$ be a $C^{\infty}$ arc in $\mathbb{R}^{2}$ with no self intersection and unit normal vector $\nu$. Then the normal derivative of $\widetilde{u}$ along $\gamma \times \mathbb{R}$ has trace $\frac{\partial \widetilde{u}}{\partial \nu} \in L_{\text {loc }}^{2}(\gamma \times \mathbb{R})$. Moreover, $B(\partial) \widetilde{u} \in L_{\text {loc }}^{2}(\gamma \times \mathbb{R})$ for every $\alpha, \beta \in \mathbb{R}$.

\section{Proof of Theorem 1.1}

Let $\Omega$ be a curved polygon, $\Omega_{\delta}$ be an open neighborhood of $\Omega$ and $T_{o}>\operatorname{diam}\left(\Omega_{\delta}\right)$. Let $E: H^{1}(\Omega) \times L^{2}(\Omega) \longrightarrow H^{1}\left(\mathbb{R}^{2}\right) \times L^{2}\left(\mathbb{R}^{2}\right)$ be a bounded linear operator which takes the pair $\left(w_{0}, w_{1}\right) \in H^{1}(\Omega) \times L^{2}(\Omega)$ to the pair $\left(\widetilde{w}_{0}, \widetilde{w}_{1}\right) \in H^{1}\left(\mathbb{R}^{2}\right) \times L^{2}\left(\mathbb{R}^{2}\right)$, where $\widetilde{w}_{0}$ and $\widetilde{w}_{1}$ are extensions of $w_{0}$ and $w_{1}$ to the plane with compact support in $\Omega_{\delta}$. Let $\widetilde{w} \in H_{l o c}^{1}\left(\mathbb{R}^{2+1}\right)$ be the solution to the Cauchy problem

$$
\begin{array}{ccl}
G(\partial) \widetilde{w}=0 & \text { in } & \mathbb{R}^{2+1}, \\
\widetilde{w}(0)=\widetilde{w}_{o} & \text { in } & \mathbb{R}^{2}, \\
\widetilde{w}_{t}(0)=\widetilde{w}_{1} & \text { in } & \mathbb{R}^{2} .
\end{array}
$$

For each $T>T_{o}$ we define the linear operator

$$
\begin{aligned}
S_{T}: H_{0}^{1}\left(\Omega_{\delta}\right) \times L^{2}\left(\Omega_{\delta}\right) & \rightarrow H^{1}\left(\mathbb{R}^{2}\right) \times L^{2}\left(\mathbb{R}^{2}\right) \\
\left(\widetilde{w}_{0}, \widetilde{w}_{1}\right) & \rightarrow\left(\widetilde{w}(\cdot, T), \widetilde{w}_{t}(\cdot, T)\right),
\end{aligned}
$$


where $\widetilde{w}$ is the solution to the problem (3.1)-(3.3). From Lemma 3 it follows that

$$
\|\widetilde{w}(\cdot, T)\|_{H^{1}\left(\Omega_{\delta}\right)}^{2}+\left\|\widetilde{w}_{t}(\cdot, T)\right\|_{L^{2}\left(\Omega_{\delta}\right)}^{2} \leq \frac{k}{T^{2}}\left\{\left\|\widetilde{w}_{0}\right\|_{H^{1}\left(\Omega_{\delta}\right)}^{2}+\left\|\widetilde{w}_{1}\right\|_{L^{2}\left(\Omega_{\delta}\right)}^{2}\right\}
$$

for every $T>T_{o}$. Now we consider the backward Cauchy problem for the operator $G(\partial)$ and take advantage of its invariance under the change of variable $t=T-\tau$. Given $\left(z_{0}, z_{1}\right) \in H_{0}^{1}\left(\Omega_{\delta}\right) \times L^{2}\left(\Omega_{\delta}\right)$, let $z \in H_{l o c}^{1}\left(\mathbb{R}^{2+1}\right)$ be the solution of

$$
\begin{array}{rll}
G(\partial) z=0 & \text { in } & \mathbb{R}^{2+1} \\
z(\cdot, T)=z_{0} & \text { in } & \mathbb{R}^{2} \\
z_{t}(\cdot, T)=z_{1} & \text { in } & \mathbb{R}^{2} .
\end{array}
$$

Applying estimate (2.14) to the function $z(\cdot, T-\tau)$ we get

$$
\|z(\cdot, 0)\|_{H^{1}\left(\Omega_{\delta}\right)}^{2}+\left\|z_{t}(\cdot, 0)\right\|_{L^{2}\left(\Omega_{\delta}\right)}^{2} \leq \frac{k}{T^{2}}\left\{\left\|z_{0}\right\|_{H^{1}\left(\Omega_{\delta}\right)}^{2}+\left\|z_{1}\right\|_{L^{2}\left(\Omega_{\delta}\right)}^{2}\right\} .
$$

Let $S_{T}^{*}$ be the bounded linear operator defined by

$$
\begin{aligned}
S_{T}^{*}: H_{0}^{1}\left(\Omega_{\delta}\right) \times L^{2}\left(\Omega_{\delta}\right) & \rightarrow H^{1}\left(\mathbb{R}^{2}\right) \times L^{2}\left(\mathbb{R}^{2}\right) \\
\left(z_{0}, z_{1}\right) & \rightarrow\left(z(\cdot, 0), z_{t}(\cdot, 0)\right),
\end{aligned}
$$

where $z$ is the solution of the problem (3.5)-(3.7).

Let $\varphi \in C_{o}^{\infty}\left(\mathbb{R}^{2}\right)$ be a test function such that $\varphi=1$ on $\Omega_{\delta / 2}$ and $\varphi=0$ in $\mathbb{R}^{2} \backslash \Omega_{\delta}$. Let $\widetilde{z} \in H_{l o c}^{1}\left(\mathbb{R}^{2+1}\right)$ be the solution of the problem (3.5)-(3.7) with $z_{o}=\varphi \widetilde{w}(\cdot, T)$ and $z_{1}=\varphi \widetilde{w}(\cdot, T)$. We have $\left(\widetilde{z}(\cdot, 0), \widetilde{z}_{t}(\cdot, 0)\right)=\left(S_{T}^{*} \varphi S_{T} E\right)\left(w_{0}, w_{1}\right)$. Define $\widetilde{v}=\widetilde{w}-\widetilde{z}$ $\in H_{l o c}^{1}\left(\mathbb{R}^{2+1}\right)$ and observe that $\widetilde{v}$ satisfies the equation $G(\partial) \widetilde{v}=0$ in $\mathbb{R}^{2+1}$ and $\widetilde{v}(T)=\widetilde{v}_{t}(T)=0$ on $\Omega_{\delta / 2}$.

Given $\left(v_{0}, v_{1}\right) \in H^{1}(\Omega) \times L^{2}(\Omega)$, we want to solve the equation

$$
\left(\widetilde{v}(\cdot, 0), \widetilde{v}_{t}(\cdot, 0)\right)=\left(w_{0}, w_{1}\right)-\left(R S_{T}^{*} \varphi S_{T} E\right)\left(w_{0}, w_{1}\right)=\left(v_{0}, v_{1}\right),
$$

where $R$ is the restriction to $\Omega$. Let us introduce the operator $K_{T}=R S_{T}^{*} \varphi S_{T} E$. In order to solve equation (3.9) we proceed as in [7] showing that $K_{T}$ is a contraction on $H^{1}(\Omega) \times L^{2}(\Omega)$ if $T>T_{o}$ is taken sufficiently large. Indeed, using the estimate (3.8) we obtain

$$
\begin{aligned}
\left\|R S_{T}^{*} \varphi S_{T} E\left(w_{0}, w_{1}\right)\right\|_{H^{1}(\Omega) \times L^{2}(\Omega)}^{2} & =\left\|\left(\widetilde{z}(\cdot, 0), \widetilde{z}_{t}(\cdot, 0)\right)\right\|_{H^{1}(\Omega) \times L^{2}(\Omega)}^{2} \\
& \leq\left\|\left(\widetilde{z}(\cdot, 0), \widetilde{z}_{t}(\cdot, 0)\right)\right\|_{H^{1}\left(\Omega_{\delta}\right) \times L^{2}\left(\Omega_{\delta}\right)}^{2} \\
& \leq \frac{k}{T^{2}} \|\left(\widetilde{z}(\cdot, T), \widetilde{z}_{t}(\cdot, T) \|_{H^{1}\left(\Omega_{\delta}\right) \times L^{2}\left(\Omega_{\delta}\right)}^{2}\right. \\
& =\frac{k}{T^{2}} \|\left(\varphi \widetilde{w}(\cdot, T), \varphi \widetilde{w}_{t}(\cdot, T) \|_{H^{1}\left(\Omega_{\delta}\right) \times L^{2}\left(\Omega_{\delta}\right)}^{2}\right. \\
& \leq \frac{\widetilde{k}}{T^{2}} \|\left(w(\cdot, T), w_{t}(\cdot, T) \|_{H^{1}\left(\Omega_{\delta}\right) \times L^{2}\left(\Omega_{\delta}\right)}^{2}\right. \\
& =\frac{\widetilde{k}}{T^{2}}\left\|S_{T} E\left(w_{0}, w_{1}\right)\right\|_{H^{1}\left(\Omega_{\delta}\right) \times L^{2}\left(\Omega_{\delta}\right)}^{2},
\end{aligned}
$$


where $\widetilde{k}$ depends on $k$ and $\varphi$. Now, using (3.4) we obtain

$$
\begin{aligned}
\frac{\widetilde{k}}{T^{2}}\left\|S_{T} E\left(w_{0}, w_{1}\right)\right\|_{H^{1}\left(\Omega_{\delta}\right) \times L^{2}\left(\Omega_{\delta}\right)}^{2} & \leq \frac{k \widetilde{k}}{T^{4}}\left\|E\left(w_{0}, w_{1}\right)\right\|_{H^{1}\left(\Omega_{\delta}\right) \times L^{2}\left(\Omega_{\delta}\right)}^{2} \\
& \leq \frac{\text { const }}{T^{4}}\left\|\left(w_{0}, w_{1}\right)\right\|_{H^{1}(\Omega) \times L^{2}(\Omega)}^{2} .
\end{aligned}
$$

where const is a convenient constant. Hence,

$$
\left\|K_{T}\left(w_{0}, w_{1}\right)\right\|_{H^{1}(\Omega) \times L^{2}(\Omega)}^{2} \leq \frac{\text { const }}{T^{4}}\left\|\left(w_{0}, w_{1}\right)\right\|_{H^{1}(\Omega) \times L^{2}(\Omega)}^{2}
$$

for every $\left(w_{0}, w_{1}\right) \in H^{1}(\Omega) \times L^{2}(\Omega)$.

Now we choose $T>T_{o}$ such that $\frac{\text { const }}{T^{4}}<1$. This makes $K_{T}$ a contraction in $H^{1}(\Omega) \times L^{2}(\Omega)$. Let $\left(w_{0}, w_{1}\right) \in H^{1}(\Omega) \times L^{2}(\Omega)$ be the unique solution to the equation (3.9). Now we define

$$
\left(\widetilde{v}_{0}, \widetilde{v}_{1}\right)=E\left(w_{0}, w_{1}\right)-\left(S_{T}^{*} \varphi S_{T} E\right)\left(w_{0}, w_{1}\right)
$$

and observe that $\left(\widetilde{v}_{0}, \widetilde{v}_{1}\right)$ is an extension of $\left(v_{0}, v_{1}\right)$ to the entire plane. The solutions of the Cauchy problems involved have the property of finite velocity of propagation and all the initial states considered have compact support. Hence, the extension $\left(\widetilde{v}_{0}, \widetilde{v}_{1}\right)$ of $\left(v_{0}, v_{1}\right)$ just defined has compact support. In the sequel we define $\widetilde{v}=$ $\widetilde{w}-\widetilde{z}$. From its construction, we see that $\widetilde{v}$ satisfies the conditions $(2.2)-(2.4)$. We can now use Lemma 1 to deduce that every pair $\left(u_{0}, u_{1}\right) \in H^{1}(\Omega) \times L^{2}(\Omega)$ has an extension $\left(\widetilde{u}_{0}, \widetilde{u}_{1}\right) \in H^{1}\left(\mathbb{R}^{2}\right) \times L^{2}\left(\mathbb{R}^{2}\right)$, with compact support such that the solution $\widetilde{u} \in H_{l o c}^{1}\left(\mathbb{R}^{2+1}\right)$ of (2.6)-(2.8) satisfies (2.9), i. e., $\widetilde{u}(T)=\widetilde{u}_{t}(T)=0$ in $\Omega$.

From Lemma 4 it follows that $B(\partial) \widetilde{u} \in L^{2}(\gamma \times[0, T])$, for each compact piece of curve $\gamma$, of class $C^{\infty}$, which is part of the boundary of $\Omega$. Hence $B(\partial) \widetilde{u} \in$ $L^{2}(\partial \Omega \times[0, T])$. Let $u \in H^{1}(\Omega \times[0, T])$ be the restriction of $\widetilde{u}$ to the cylinder $\Omega \times[0, T]$ and define $g=B(\partial) \widetilde{u}$. It follows from the construction above that $T>0$, $u$ and $g$ satisfy the conditions (1.2)-(1.5) of Theorem 1 .

\section{Proof of Theorem $\mathbf{1 . 2}$}

Let $\Omega$ be a curved polygon contained in the interior of the sector $\Omega_{\infty}$ as in section 1. We denote $V(\Omega)$ the closed subspace of $H^{1}(\Omega)$ of the functions vanishing on $\Gamma_{0}$ (the sides of $\Omega$ laying on the edges of $\Omega_{\infty}$ ). From the assumptions on the boundary of $\Omega$ it follows that each function $w \in V(\Omega)$ has an extension $\widehat{w} \in H_{0}^{1}(\widehat{\Omega})$ where $\widehat{\Omega}$ is a smooth domain satisfying $\Omega \subset \widehat{\Omega} \subset \Omega_{\infty}$ and $0<a<|x|<b<\infty$ for every $x \in \widehat{\Omega}$ (see [9], p. 131). Such function has an extension $\widetilde{w}$ to the entire $\mathbb{R}^{2}$ as odd function with respect to each line $\theta=i \frac{\pi}{m}, i=0, \ldots, m-1$, such that $\widetilde{w} \in H^{1}\left(\mathbb{R}^{2}\right)$ and supp $\widetilde{w} \subset U=\left\{x \in \mathbb{R}^{2}: a<|x|<b\right\}$. Moreover, there exists a sequence $\widetilde{w}_{n} \in C_{0}^{\infty}\left(\mathbb{R}^{2}\right)$ such that $\widetilde{w}_{n}$ is odd function with respect to each line $\theta=i \frac{\pi}{m}, i=0, \ldots, m-1$, supp $\widetilde{w}_{n} \subset U$ and $\widetilde{w}_{n}$ converges to $\widetilde{w}$ in $H^{1}\left(\mathbb{R}^{2}\right)$. If $w \in L^{2}(\Omega)$, an extension $\widetilde{w} \in L^{2}\left(\mathbb{R}^{2}\right)$, odd with respect to the lines $\theta=i \frac{\pi}{m}, i=0, \ldots, m-1$, and supported in $U$, can be constructed extending by zero to the sector $\Omega_{\infty}$ and then by reflecting on each line 
$\theta=i \frac{\pi}{m}$. Hence, there exists a bounded linear operator $E$ which extends each pair $\left(w_{0}, w_{1}\right) \in V(\Omega) \times L^{2}(\Omega)$ to the pair $\left(\widetilde{w}_{0}, \widetilde{w}_{1}\right) \in H^{1}\left(\mathbb{R}^{2}\right) \times L^{2}\left(\mathbb{R}^{2}\right)$, where $\widetilde{w}_{0}$ and $\widetilde{w}_{1}$ are odd with respect to the lines $\theta=i \frac{\pi}{m}, i=0, \ldots, m-1$ and have compact support in $U$. Now we proceed as in the proof of Theorem 1. First we set $T_{o}>b(=\operatorname{diam}(U))$ and define for each $T>T_{o}$ the linear operator

$$
\begin{aligned}
S_{T}: H_{0}^{1}(U) \times L^{2}(U) & \rightarrow H^{1}\left(\mathbb{R}^{2}\right) \times L^{2}\left(\mathbb{R}^{2}\right) \\
\left(\widetilde{w}_{0}, \widetilde{w}_{1}\right) & \rightarrow\left(\widetilde{w}(\cdot, T), \widetilde{w}_{t}(\cdot, T)\right),
\end{aligned}
$$

where $\widetilde{w}$ solves the problem (3.1)-(3.3). We observe that $\widetilde{w}$ vanishes on the planes of $\mathbb{R}^{2+1}$ through the lines $\theta=i \frac{\pi}{m}, i=0, \ldots, m-1$, and parallel to the $t$-axis. This follows from the explicit formulas (2.11) and (2.12) for the solution $\widetilde{w}$ and the mentioned oddness of $\widetilde{w}_{0}$ and $\widetilde{w}_{1}$. From $(2.11)$ and $(2.12)$ it also follows that $\widetilde{w}(\cdot, T)$ and $\widetilde{w}_{t}(\cdot, T)$ are odd with respect to the lines $\theta=i \frac{\pi}{m}, i=0, \ldots, m-1$ vanishing on them.

The cut off function $\varphi \in C_{o}^{\infty}\left(\mathbb{R}^{2}\right)$ now is taken to be radial, supported in $U$ and being $\varphi=1$ on the set $\left\{x \in \mathbb{R}^{2}: a+\delta<|x|<b-\delta\right\}$ for a convenient $\delta>0$. The rest of the proof is the same as for Theorem 1. To finish we just observe that function $\widetilde{u} \in H_{l o c}^{1}\left(\mathbb{R}^{2+1}\right)$, constructed in the same fashion as in Theorem 1, vanishes on the faces of $\Omega_{\infty} \times \mathbb{R}$.

\section{Concluding Remarks}

Remark 1: In theorems 1 and 2, the time $T_{o}$ is large enough to allow every ray of the geometric optics propagating inside $\Omega$ to reach the support of the control function before $T_{o}$ has elapsed. In other words, the geometric control condition established in [1] is verified for the problems considered here.

Remark 2: Most of the results available on control with mixed boundary condition demands $\Gamma_{o}$ to be $\left\{x \in \partial \Omega:\left(x-x_{o}\right) \nu(x) \leq 0\right\}$, for some $x_{o}$. The case considered in Theorem 2 may not fit this pattern. Observe that for $\Omega=\Omega_{\infty} \cap\{x: 0<a<|x|<b\}$ there is no $x_{o}$ such that the $\Gamma_{o}$ is exactly the set $\left\{x \in \partial \Omega:\left(x-x_{o}\right) \nu(x) \leq 0\right\}$.

Remark 3: When the type of boundary condition changes, singularities can appear in the solution of mixed problems. This is one of the difficulties in dealing with the control problem with mixed boundary conditions (see [3]). Fortunately, the approach we used here kept us far from that kind of trouble.

Resumo. Neste trabalho estudamos controle exato na fronteira para uma equação diferencial parcial linear, de segunda ordem, com coeficientes constantes, cuja parte principal é o d'Alembertiano. Consideramos domínios do plano, com fronteira suave por partes, dados iniciais com energia finita e controle do tipo Robin, atuando em toda a fronteira ou somente em uma parte dela. 


\section{References}

[1] C. Bardos, G. Lebeau, J. Rauch, Sharp sufficient conditions for the observation, control and stabilization of waves from the boundary. SIAM J. Control Optim., 30 (1992), 1024-1065.

[2] W.D. Bastos, A. Spezamiglio, A note on the controllability for the wave equation on nonsmooth plane domains. Systems Control Lett. 55 (2006), 17-20.

[3] P. Grisvard, Controlabilité exacte des solutions de l 'equation des ondes en présence de singularités. J. Math. Pures Appl., 68 (1989), 215-259.

[4] J.-L. Lions, Contrôlabilité exacte des systèmes distribués. C. R. Math. Acad. Sci. Paris, Ser. I Math., 302 (1986), 471-475.

[5] W. Littman, Near Optimal Time Boundary Controllability for a Class of Hyperbolic Equations, In "Lecture Notes in Control and Inform. Sci.", p.306-312, Springer, New York, 1987.

[6] W. Littman, Remarks on Boundary Control for Polyhedral Domains and Related Results, in "Boundary Control and Boundary Variation", (J.P. Zolézio, ed.), p.272-284, Springer, New York, 1990.

[7] D.L. Russell, A unified boundary controllability theory for hyperbolic and parabolic partial differential equations, Stud. Appl. Math., 52 (1973), 189-211.

[8] D. Tataru, On the regularity of boundary traces for the wave equation. Ann. Sc. Norm. Super. Pisa Cl. Sci. (4), 26 (1998), 185-206.

[9] J. Wrokla, "Partial Differential Equations", Cambridge University Press, 1987. 